\title{
COMPLETELY INTEGRATED THERMO-ELECTRO-MECHANICAL ANALYSIS OF MEMS DEVICES
}

\author{
Sandeep Akkaraju, Yie He, Robert Harris, Gene Napadensky, and Fariborz Maseeh \\ IntelliSense Corporation \\ 16 Upton Drive \\ Wilmington MA 01887 \\ E-mail: IntelliS@aol.com
}

\begin{abstract}
In this paper we describe the development of a CAD tool for performing completely integrated thermo-electro-mechanical analysis for MEMS devices. As a demonstration of the capability, we analyze the performance of an electrostatic actuator under applied temperature, voltage, residual stress and mechanical loading.

\section{INTRODUCTION}

Temperature related issues such as sensitivity, residual stresses and CTE mismatch are important issues in translating MEMS devices into commercial instruments. High temperature processing of micromachined devices such as anodic bonding and doping processes during the fabrication of on-chip electronics can induce significant temperature artifacts in the device performance. Additional process associated effects such as warpage caused by stress gradients in deposited films, process tolerances add to the difficulty of designing MEMS devices. Lack of proper analysis tools have further impeded the successful design and development of micromachined devices and systems.

IntelliSense has been actively involved in developing CAD tools for the MEMS community. We have previously reported the development of a virtual prototyping environment (IntelliCAD ${ }^{T M}$ ) for designing MEMS [1]. In this paper, we report a key improvement to the IntelliCAD software suite to perform fully integrated thermo-electro-mechanical analysis. The software system allows the user to account for process induced and operating environment effects for the accurate analysis of the real time performance of microdevices.
\end{abstract}

\section{THEORY}

Fully coupled thermo-electro-mechanical analysis is a computationally difficult problem. First, the problem requires mesh compatibility between the electrostatic analysis (usually, boundary element based) engine and the mechanical analysis (usually, finite element or finite difference based) engine. Electrostatic analysis requires the presence of fine mesh around sharp edges or comers where the charge density is concentrated, while the mechanical analysis requires the mesh to be refined near large stress or stress gradient regions.

Second, the discretization grid must track the electrostatically deformed boundaries of the structure--i.e., solve a moving boundary problem. The problem is further complicated by requirements such as automatic or adaptive generation of optimized meshes of structures.

The governing system of equations for a thermoelectromechanical problem can implicitly be expressed as:

$$
S=F_{m}\left[X_{0}(T, M), F_{e}\left(S+X_{0}, V\right)\right]
$$

where: $X_{0}(T, M)$ is a vector representing the released structure, $S$ is the discretized structure surface displacement, $T$ is the applied thermal boundary, $M$ is the multi-material property information and $V$ is the applied voltage information. $F_{e}$ represents the electrostatic force derived from solving the Laplace equation. The final displacement, $S$, can be obtained by solving the forcedisplacement equation.

\section{IMPLEMENTATION}

The central algorithm for solving coupled thermoelectromechanical problems is described below. First, the IntelliCAD finite element program is used to perform true 3D thermal analysis to determine the temperature distribution, this is followed by the calculation of thermal stress gradients and process induced effects to simulate the released device structure. In the next step, the voltage loads are applied and the electrostatic pressure distribution is calculated using the IntelliCAD 3D electrostatic module. The electrostatic pressure loads are then applied in the finite element mechanical engine and the deformations are recalculated. This procedure is repeated until a preset convergence criterion is reached. This method will converge if:

$$
\left|\frac{\partial F_{m}}{\partial F_{e}} \cdot \frac{\partial F_{e}}{\partial S}\right|<1
$$

In case of divergence due to electrostatic instability such as pull-in of structures the analysis is terminated.

\section{EXAMPLE}

In order to demonstrate the coupled analysis we analyzed a surface micro-machined linear resonating actuator fabricated using the MCNC-MUMPS process [2]. The mask-set (6 masks) used for the device fabrication was obtained from the MCNC MEMS webpage [3].

Figure 1 shows the IntelliCAD solid model of the surface micromachined device (the vertical scale has been exaggerated for the purpose of clarity). The solid model shows the presence of anchors, dimples and the poly interconnect features (poly0, dimple, anchor1, poly1, poly1_poly2_via, hole1, etc.).

For the purpose of demonstration, we assumed that the substrate is heated while the resonator is maintained at room temperature. A potential difference of $20 \mathrm{~V}$ was applied between the fixed comb and the actuator. We also accounted for the presence of process induced stresses in the deposition of the polysilicon and subsequent doping [4].

\section{RESULTS AND CONCLUSIONS}

Figure 2 shows the deformation of the device due to the process induced stresses, operating temperature effects and applied voltage loads - the result of the fully coupled thermo-electromechanical analysis. Figure 3 shows a close-up of deformed structure, the warpage induced effects are clearly visible.

The fully coupled analysis has been successfully implemented using the IntelliCAD architecture. The module can successfully handle multi-dielectric effects. The IntelliCAD system. seamlessly integrates MEMS process design, device mask layout and fully coupled thermo-electro-mechanical analysis of micromachined devices. The efficacy of the IntelliCAD system has been tested in designing a wide range of micromachined sensors and transducers. This includes -- micromachined pressure transducers, microphones and resonating gyro elements. 


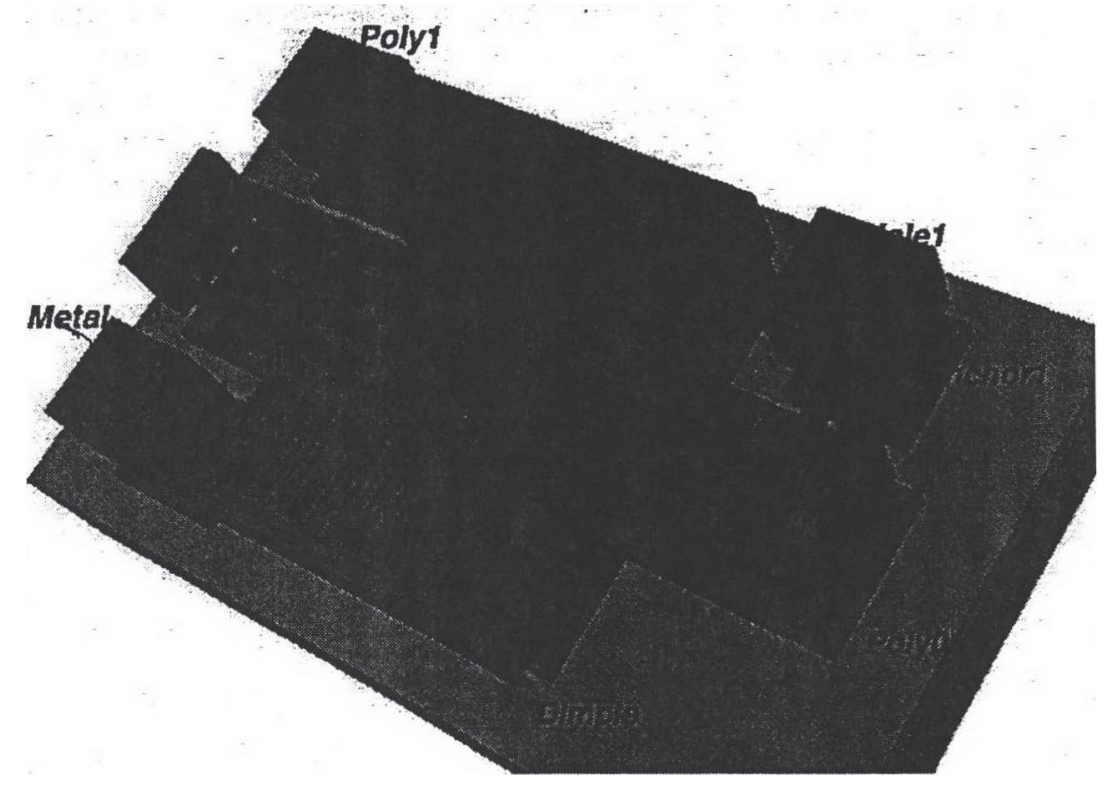

Figure 1. Solid model of a surface micromicromachined linear actuator generated by IntelliCAD using a mask set provided by MCNC.

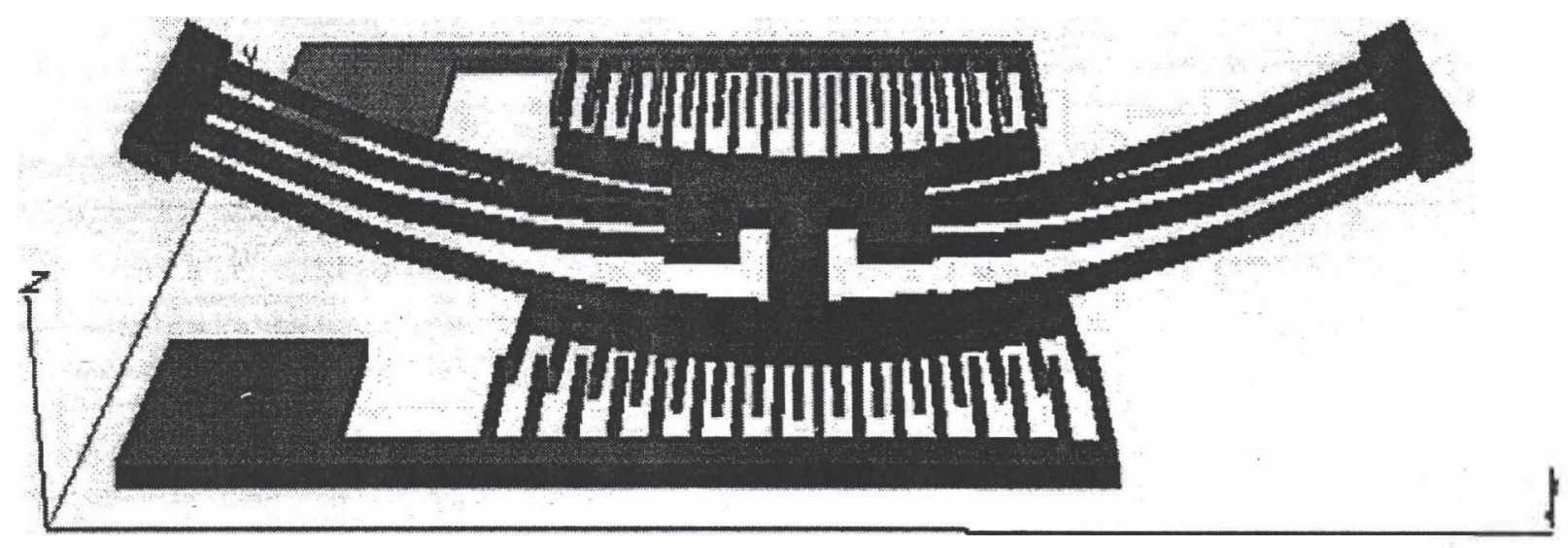

Figure 2. Warpage of the polysilicon structure due to process related residual stresses, thermal loading effects and voltage loads. The finite element analysis was performed using 1169, 20 noded iso parametiic brick elements. The deformation of the structure has been exaggerated for the purpose of clarity.

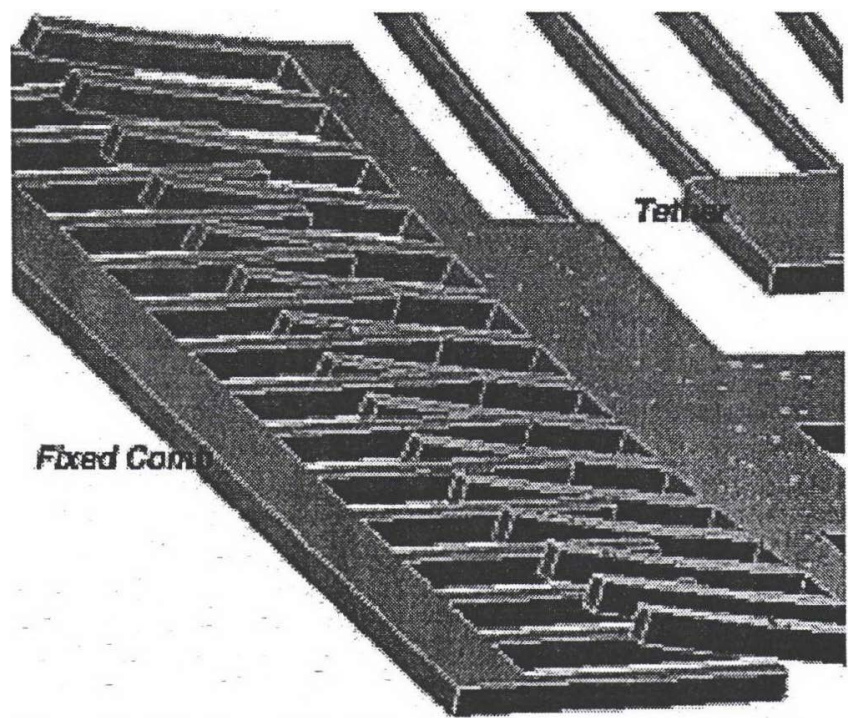

Figure 3. Close-up of the deformation of the comb-teeth. (Deformation has been exaggerated for the purpose of clarity)

\section{ACKNOWLEDGEMENT}

The development of IntelliCAD is partially sponsored by the Office of Naval Research SBIR program.

\section{REFERENCES}

1. Y. He, R. Harris, G. Napadensky and F. Maseeh, "A virtual prototype manufacturing software system for MEMS", Proceedings of IEEE MEMS '96 (1996)

2. K. W. Markus and D. A. Koester, "Multi-User MEMS Process (MUMPS) - Introduction and Design Rules", Published by MCNC, Electronics Technologies Division (1995)

3. Nonparametrized Microelectromechanical Library, http://mems.mcnc.org/cug/mc5.html also see the MCNC -MEMS Internet homepage, http://mems.mcnc.org/mumps/ (1996)

4. IntelliSense Corporation, MEMaterial@ - Solid state thin film database - Properties of thinfilm polysilicon (1993-1996) 